\title{
Taking Participatory Citizen Science to Extremes
}

\author{
Matthias Stevens, Michalis Vitos, Julia Altenbuchner, Gillian Conquest, Jerome Lewis \& Muki Haklay \\ Extreme Citizen Science (ExCiteS) research group, University College London \\ Gower Street, London, United Kingdom
}

\{m.stevens, michalis.vitos.11, julia.altenbuchner.11, g.conquest.11, jerome.lewis, m.haklay\}@ucl.ac.uk

\begin{abstract}
UCL's Extreme Citizen Science (ExCiteS) research group is experimenting with ways to incorporate the most marginalised communities into participatory citizen science activities through which they can share their indigenous knowledge. We work with communities at the extremes of the globalised world - both because of non-literacy and the remote or forbidding environments they inhabit. These groups are the gatekeepers of some key environments on which the future health of the planet depends - from tropical forests to Arctic sea-ice. Here we present the methodologies and tools we are developing to give them a voice.
\end{abstract}

\section{INTRODUCTION}

Information and Communication Technologies (ICTs) are central to the 'flattening' of today's globalised world. While the rise of ubiquitous and pervasive computing applications facilitates interaction and information sharing between individuals, there has not been a parallel breakthrough in applications that help communities work together to solve common problems in a way that supports action towards sustainability. Citizen science is one of the most innovative areas seeking to achieve this.

Participatory citizen science offers an innovative, promising solution to achieve long-term sustainable management of key world environments and greater respect for the rights of those living in them by empowering them to collect, interpret and use scientific information in a way that is useful for them [1]. To further develop participatory citizen science, UCL's ExCiteS group has taken on the challenge to develop both methodologies and tools enabling wider participation by lay people, especially those with limited technical abilities and limited literacy, living in extreme environments. We work with marginalised groups, such as indigenous peoples, to support them to combine scientifically sound methods with local knowledge so they can participate more effectively in decision making processes relating to pressing issues such as deforestation, biodiversity loss and food security.

This paper introduces the key elements of our approach to expand the reach of citizen science. Our approach, called 'Extreme' Citizen Science, is supported by a methodological and a technological pillar. The methodological pillar is based on community engagement protocol designed around a free, prior and informed consent (FPIC) process [2] and Participatory Design [3], [4]. The technological pillar is formed by Sapelli, a new mobile data collection platform designed to be equally accessible to both non-literate and literate users, and to allow data transmission in extreme environments. To illustrate these, we describe some of our experiences in on-going work involving communities with little or no formal education nor ICT experience.

If citizen science is to fulfil its promise of stimulating public interest, participation and representation in scientific research, and the policies that research is expect to inform, then it is important that all citizens, especially those that are the hardest to reach due to geographical, cultural, political, educational or socio-economic reasons, can, at least in principle, be offered a chance to participate and benefit. Similarly, if pervasive computing is to succeed in making computing a truly ubiquitous force for good in our societies, then it is important to look beyond the urban jungles in which most technology reside and come up with technologies that work even in the most extreme circumstances. Therefore, although our current focus is mainly on marginalised, ignored groups living in remote, unconnected places, we believe that the tools and methodologies presented here hold relevance, both in combination and in isolation, to the broader citizen science and pervasive computing communities.

\section{From Citizen to PARTICIPATORY SCIENCE}

Citizen science is typically understood as scientific activities - usually the collection and sometimes processing of data - carried out by non-professional scientists in the context of a scientific project. Citizen science, has a very long history, but has gained recognition and attention in recent years [5], [6]. Important driving forces are the proliferation of ICT in general and pervasive computing in particular; the realisation that the public can provide free labour, skills, computing power and even funding (cf. crowdsourcing and crowdfunding); and the growing demands from research funders for public engagement. As a result, the last decade has seen the of rise of new, ICT-enabled incarnations and interpretations of the concept and an explosion of new citizen science projects. 
However, the majority of such projects are set in developed countries and usually participants are expected to have some formal education and familiarity with ICT before joining the activity. Often the involvement of participating 'citizen scientists' is limited to specific phases of the process. For instance, participants may be viewed as sensors [7] or data collectors [6] but are rarely invited to decide what data to collect, analyse data or contribute to its interpretation, although having carried out the primary observations they may have valuable insights. Bonney et al. [5] identify three types of citizen science projects - contributory: participants contribute data to scientific research; collaborative: scientists design the project, members of the public are involved in refining it or analysing data; and co-created: scientists and the public work together. A recent review of environmental citizen science projects in the UK demonstrated that only a small fraction are co-created [8].

Meanwhile, practitioners of ICT for Development (ICT4D) [9], Participatory Rural Appraisal (PRA) and Participatory Action Research (PAR) [10], and Participatory Geographic Information Systems (PGIS) [11] highlight the importance of participatory and inclusionary methods to ensure that local communities' needs and knowledge are well represented.

Therefore, we seek to combine the power of ICT with lessons from participatory and inclusionary methods. Developing new tools and the methodologies through which they can be successfully deployed, requires an interdisciplinary approach. Anthropological methods help to understand local contexts and to identify and engage with potential users. Human ecology, geography and development studies allow to diagnose the challenges these communities face, while ICT4D offers models for capitalising on the rapid spread of communications technologies. Insights from Human-Computer Interaction (HCI) inform the design of data collection, mapping and visualisation tools, and pervasive computing and software engineering are integral to implementing these. By working across disciplines we seek to stretch existing citizen science practice and extend its scope to tackle issues to which it is well-suited, but is rarely applied.

\section{Methodology}

We aim to enable marginalised communities to participate in and benefit from citizen science and the mobile technology that facilitates it. Yet, deploying technology and offering people the opportunity to take part in citizen science activities is not enough to empower marginalised communities. Typical pitfalls include cultural misunderstandings, inappropriate technology, misinterpretations of the purpose of engagement, inflated expectations, misreadings of power dynamics, ineffective or divisive incentives, and various organisational issues. All can lead to disinterest, disappointment, conflict, unintended consequences and even failure. We do not claim to have resolved these issues, but we are trying. Rather we want to point out that in order for ICT-enabled citizen science initiatives to reach out to marginalised groups these issues must be faced clearly and honestly. To have more chance of positive outcomes, projects must be framed in carefully designed protocols adapted to the specific geographical, cultural, political, educational or socio-economic context, and which are flexible enough to deal with changing circumstances. Moreover, if citizen science is to empower communities it is important that protocols stimulate co-creation and inclusion, rather than only seeking contribution.

Below we outline solutions we have developed to overcome some of the mentioned issues above. These draw upon anthropological research and lessons learned during participatory monitoring projects involving nonliterate indigenous communities in Central-Africa [2], [3] - see section 4 .

\subsection{Intermediaries}

Due to the way funding is obtained, in many cases the initiative to set up a participatory citizen science project is taken by actors - such as non-governmental organisations (NGOs) or academics - who are very distant from targeted participant communities. To counter the risks this poses, sufficient expertise about local conditions and a strong presence in the target region are required. Partnerships with intermediaries already working closely with, and trusted by participating communities, and who are able to manage the project locally are crucial. They give the project legitimacy to participants and other local stakeholders. Examples of such intermediaries in our projects are local authorities, international and local NGOs, and representatives of participating communities.

\subsection{Community engagement protocol}

What follows is a protocol we apply from the first contact with a community of potential participants.

Whenever possible we announce our visit a day or two before coming by presenting the project to local authorities or elders to ask for permission to proceed with a general assembly of the community. We seek to ensure that the assembly being consulted is representative of the diversity of the larger community - i.e. involving both men and women, young and old, and different ethnic backgrounds. If potential participants span numerous communities we repeat the protocol in multiple localities ensure cross-community agreement on concepts and representations.

Once all assembled, we initiate a process of free, prior and informed consent (FPIC) [2]. This begins by thoroughly introducing ourselves and any other stakeholders. We explain, in broad terms, the purpose of the project, the potential role for the community; and then explain what we understand to be the associated risks and benefits. We encourage discussion and ask questions to gauge the extent to which key issues have been understood and debated. In the context of environmental justice projects we 
spend a lot of time co-developing with the community effective advocacy strategies and partnerships. We never assume the community's willingness to participate, and will only ask them explicitly to give their consent once we are satisfied that the issues are understood by an inclusive majority. The terms of participation are left open for discussion and we take care to avoid unrealistic expectations on either side. We emphasise that they can withdraw their consent at any time.

If the community expresses an interest in participating, the next phase is an exercise in co-creation and iterative participatory design [4]. Having understood the purpose of our collaboration, participants now contribute to developing the data collection interface. The first step is to define the types of information to be collected. Working with a prototype, the key measurements, environmental parameters or local observations to be made are discussed. Participants comment on their ability and willingness to provide the information (e.g. observations of illegal activities can have consequences), and whether they consider it relevant to do so. We stimulate people to suggest other or additional types of information they consider important. During this discussion a commonly understood set of concepts and representations thereof (e.g. terminology or iconography) are established to structure collecting and visualising of data later on. We refrain from introducing technology during this conceptual phase so as not to distract or confuse people. In projects involving with non-literate users concepts will likely be represented by graphical icons. Big flashcards are used to allow a large crowd to guess the concept being represented - making the exercise fun, participatory and accessible. In all cases feedback is carefully noted and incorporated into the interface prototype before visiting the next community to ensure an iterative process and incorporation of community views.

Following this design phase we introduce and demonstrate the tools that will be used. These may include hardware (e.g. GPS receivers, smartphones, measuring equipment, etc.) as well as software. To teach participants to use them training needs to be adapted to users' abilities. Following the demonstration we let users freely interact with the tools while remaining on hand to observe and help out. The duration of this familiarisation stage varies, but users should be given adequate time to explore the tools' affordances and to gain confidence in using them. Fast learners are encouraged to support others. When users feel comfortable with the tools, their understanding is tested by requests to carry out small tasks. Meanwhile we provide constant feedback to those who find it hard. Finally, we contextualise the activities by asking users to apply what they have learned in more realistic exercises in the local environment. For instance, in community resource mapping projects the final step of the training is to ask small groups to spend a specified amount of time (e.g. two hours) touring the area while mapping resources. During this contextualised training we tend to stay mostly silent and intervene only when asked. Throughout the whole training process users' comments and suggestions regarding the tools, the interaction or the process are carefully noted to guide further improvements.

Finally, in view of the likely benefits and the potential risks involved in any future deployment, we ask whether, and under what conditions, community members would be willing to participate in a longer term deployment. If they are interested, further extended discussions are organised to construct an engagement protocol between community members, the project and other local stakeholders. Key areas for negotiation are remuneration strategies and access to the collected data. The community decides what, with who, and to what extent to share their data.

\section{Case studies}

Our approach was developed mostly in collaborations with indigenous communities in the Congo Basin rainforest. Some of these communities' are semi-nomadic hunter-gatherers (Pygmies), others are sedentary farmers, but all crucially depend on the forest for their livelihoods. They are among the poorest African citizens, yet they are rarely involved in the management of the areas on which they depend. Addressing the needs of these groups is challenging on many levels: Local infrastructure is weak or non-existent; Governance is similarly weak and undermined by corruption and resource-fuelled conflict; Economies are dominated by multinationals extracting oil, minerals and timber, and increasingly promoting large scale land-use change by establishing palm oil plantations. Climate change is a new, unpredictable factor with local and regional implications, and current conservation and natural resource management efforts often involve draconian measures that disenfranchise forest people.

In 2005, Lewis et al. established a scheme for forest people to play an active role in the monitoring of logging activity. The Mbendjele, hunter-gatherers living in Congo-Brazzaville, helped develop bespoke software that allowed these non-literate users to record their resources, and violations thereof, using a pictorial decision tree running on a rugged personal digital assistance (PDA) device. This information was used by the local logging company to comply with Forest Stewardship Council principles to respect local peoples' resources. In 2007 an initiative to monitor illegal logging was set up in Cameroon [2], [3].

In 2010 Mbendjele involved in the first project requested Lewis to set-up a similar scheme to deal with another pressing issue: commercial poaching. Expanding logging roads and the highly lucrative ivory trade have led to a rapid expansion in poaching. This is problematic for forest people because of over-hunting and reprisals made against them by government-run 'eco-guards', supposedly responsible for controlling poachers, but often looking for easier targets. In 2012 the newly-formed 
ExCiteS group took up this challenge and developed a prototype of an 'Anti-Poaching' application, based on the Open Data Kit (ODK) platform [12]. This app lets participants record evidence of poaching activity (geolocated via GPS and optionally augmented with photos and/or audio) using a pictorial decision tree containing icons co-designed with Mbendjele representatives [13]. This project is ongoing but the ODK-based app has been replaced by one built on our new Sapelli platform.

Also in 2012, we partnered with Forests Monitor, an international NGO and CAGDF, a local forestry sector watchdog in Congo-Brazzaville, to develop ways to enable forest people to monitor the legality and socioeconomic impacts of logging activities. The project enables locals to give direct feedback on the behaviour of logging companies, and allow them to accurately map their key resources to protect them from destruction. All observations are made using a bespoke pictorial decision tree, see figure 1 based on the Sapelli platform.

Together with another international NGO, Forest Peoples Programme, we are developing tools to better support community engagement in a REDD $+^{1}$ monitoring project in the Democratic Republic of Congo. In Ethiopia our tools support research investigating how traditional agro-pastoralist communities are adapting to rapid changes in their environment and new land-use laws. In the Brazilian Amazon we are working with indigenous peoples to develop land management tools. In the Arctic we are working with Iupiat walrus hunters worried about climate change by helping them to monitor sea-ice change and share data with experts at NASA.

In each of these initiatives the goal is to build solutions, in collaboration with local experts and participating communities, to promote indigenous peoples' control of their land and resources. The overarching approach is to introduce bespoke tools - built on top of the same underlying platform - that allow participants to capture local (environmental) knowledge, report insitu observations, visualise and discuss results, and share data with selected outsiders.

\section{SAPELLI DATA COLLECTION PLATFORM}

In recent years a growing range of mobile data collection platforms and services have emerged. The first generation of platforms, like CyberTracker ${ }^{2}$, targeted PDAs and now feels outdated. This was followed by a new crop of smartphone-based platforms, like EpiCollect [14] and ODK [12]. We evaluated these in terms of our needs, with specific attention to survey design and data synchronisation. All support the creation of sequential survey forms and some even support icon-driven surveys. However none are entirely text-free, which is problematic for users with low literacy. All reviewed platforms allow data collection while offline, postponing data transmission to

1. United Nations Programme on Reducing Emissions from Deforestation and Forest Degradation.

2. http://cybertracker.org. a later stage. However, these systems rely on Internet connection and typically require user action to start the synchronisation process. This is problematic when network connectivity is rare, unstable, slow or expensive, especially if users lack phone experience. In such cases it is desirable for tools to be 'smart' enough to autonomously detect synchronisation opportunities and choose between alternatives (including SMS) depending on availability, bandwidth, cost, etc.

In 2012 we used ODK to build the 'Anti-Poaching' application prototype (see section 4 ). We had to make extensive changes in the ODK code, notably to remove all textual and numerical UI elements [13]. The main problem, however, was the unsuitability of ODK's XForms-based survey description format for modelling hierarchical flows. Implementing the decision tree required extremely verbose and complicated XML code, constraining readability, evolution and reuse.

After evaluating other alternatives we found that none of them met our requirements, particularly with regards to text-free, hierarchical interfaces, and autonomous multi-modal synchronisation. Therefore, since late 2012 we have been developing a new data collection and transmission system from scratch. The Sapelli data collection platform - named after the endangered sapelli tree (Entandrophragma cylindricum) which is important to forest people as a source of caterpillars - is still a work in progress but it has already undergone three field trials (see section 6). A beta version can be found at http:/ / sapelli.org.

Sapelli currently consists of 3 main components. The Sapelli Collector app is our data collection and transmission client for Android devices. The Sapelli SMS Relay, an app responsible for forwarding data sent to it via SMS by Collector instances. Finally there is a - currently still rudimentary - server application to handle centralised reception, data storage and report generation in various formats.

\subsection{Survey Design}

A principal aspect which sets Sapelli apart from most other mobile data collection platforms is our focus on low- and non-literate users. Concretely we allow the design of text-free surveys based on touch-interaction with pictorial decision trees. These surveys allow users to collect georeferenced data, pictures, audio recordings through an interface that are devoid of any textual or numerical elements. It is important to note that the utility of pictorial interfaces goes well beyond non-literate or illiterate users. We expect them to be equally appropriate in data collection projects involving young children, the elderly, and people with bad eyesight or other disabilities. More generally, there are various context in which icon-driven interfaces are more practical, faster or efficient for literate users as well.

Sapelli also supports conventional textual forms containing widgets like checkboxes, text fields, etc. Pictorial and textual forms can be harmoniously integrated 
with clear boundaries and possibly access restrictions between them. This is useful in cases where users with different abilities and/or roles need to use the same device. For instance, NGO representatives can set up monitoring sessions using a textual form, after which the same device can be passed on to non-literate community members to collect data associated with that session.

Unsatisfied with overly complicated existing survey description formats such as XForms, we designed our own XML-based format. Sapelli XML provides a set of predefined building blocks called fields (e.g. $\langle$ Choice $\rangle$,

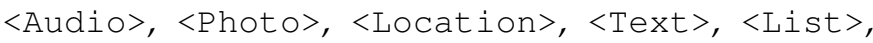
etc.) to describe surveys consisting of one or more forms. Because our methodology calls for rapid, in-situ adaptations of data collection interfaces we have kept the format as simple and concise as possible. Ideally modest computing skills should suffice to quickly learn how to create and update surveys.

Figure 2 shows an example of how a simple pictorial survey can be described in Sapelli XML and how it would appear in the screen. The description of a form not only defines how it will appear on the screen but also how data entries are stored. Upon parsing the XML a database schema is inferred in which each form field is mapped onto a column.

Decision trees are built by nesting <Choice> nodes. This makes the structure of the decision space (i.e. the tree) immediately apparent by looking at the code. It is interesting to note that such conditional constructs can not only be used to create classification hierarchies, such as the "TransportNode" tree in the example, but also to direct control flow, as with the "Confirmation" and "Next" fields.

\subsection{Data synchronisation}

Sapelli provides an autonomous, multi-modal data transmission mechanism to submit survey entries to a central server. The Collector app includes a background service that automatically checks for connectivity at scheduled intervals. In order to conserve power it can optionally put the device into flight mode between checks. When there is data to be sent, and a transmission opportunity arises, the service autonomously decides what to transmit and how, depending on available networks, bandwidth and project-specific settings.

The transmission of basic form entry data (i.e. timestamps, decision tree selections, coordinates, text input, etc.), which requires little bandwidth, happens independently of larger chunks like photos and audio recordings. This is useful in cases where urgency and timeliness is a factor. To transmit basic data, records are serialised in a binary format which is heavily optimised for space. Next, these are grouped in transmissions that can either be sent in up to 16 chained SMS messages, or via HTTP (over cellular or Wi-Fi networks). Transmission payloads are compressed and can be encrypted. SMS messages are sent to phone running the SMS Relay, which forwards them to the server. A hashing algorithm ensures that attachments can be reliably re-associated with corresponding records.

\subsection{Sapelli Launcher}

Of course users who require a text-free survey app are likely to have difficulty when dealing with Android's text-heavy interface. Therefore we have developed the Sapelli Launcher app, which replaces the standard Android UI with a restricted, text-free version that only shows icons for a set of allowed apps. These can be tailored to project requirements and user abilities. To prevent unauthorised access, apps can be protected with a mechanism similar to Android's pattern unlock feature.

\section{Evaluation}

In the Spring of 2013, an ExCiteS delegation travelled to northern Congo-Brazzaville for 6 weeks to field test the Sapelli platform, and evaluate and improve our methodology. Working closely with representatives of Forests Monitor and CAGDF, we visited eight villages and camps, and the base camps of three logging companies in whose concessions the field sites are situated. The sites were chosen to allow gathering feedback from a range of contexts: we worked with both farmer and hunter-gatherer communities, across sites of differing remoteness from urban centres (and therefore likelihood of familiarity with ICT), and in concessions run by logging companies with different approaches to their environmental and social obligations.

In every field site we followed the methodology presented in section 3. During the training sessions Sapelli Collector was introduced to a total of 276 participants (146 male, 130 female) and 138 participants (80 male, 58 female) took part in subsequent mapping exercises. In each site enthusiasm for the project was strong and participants expressed the desire for a longer-term deployment. We encountered some technical challenges such as battery life, or inconsistent performance of local cellular networks. However, our focus in this section is to share the interaction challenges we identified when observing participants using our software.

Across all field sites we found that some people developed proficiency with the software very quickly, while others required much more training, still requiring assistance to navigate the UI even after the mapping exercise. Rapid proficiency was unrelated to any specific factors we could identify - it occurred among both Bantu and Pygmy groups, was both among non-literate and schooled individuals, among men and women, and within both the more remote and the better-connected sites. While many ICT4D practitioners have emphasised that young people are usually quickest to assimilate the "aural and visual cues and metaphors in a welldesigned computer interface" [9, pp.332], we observed no particular age bias among our rural users - often 
it was middle-aged and older members of a community helping younger members to understand how the software worked, and vice versa. We plan to capitalise on this observation by developing peer-to-peer training strategies to scale-up software introduction for larger project sites by employing the most proficient users from a satellite village as "infomediaries" [9, pp.336].

Most users easily recognised icons, but interface navigation and the understanding of certain icons was hampered by a lack of familiarity with common symbolic or metaphorical conventions like arrows, crosses, ticks, the use of green and red to respectively signify positive and negative conditions. At a more general level, many participants seemed to have trouble grasping the overall hierarchical structure and how to navigate through it using forward and backward steps. We also saw indications of a possible correlation between the amount of trouble users had and the depth of the hierarchy. These anecdotal observations may be explained by the research of Medhi et al., who point out that, because low literacy levels are usually the result of a lack of (formal) education, non-literates may also struggle with cognitive abilities such as conceptual abstraction and categorization - which may explain difficulty in navigating hierarchical, yet text-free UIs [15]. More research is needed in this area.

Touchscreen interaction proved to be challenging for some users who were uncertain how long they needed to press an icon for the device to register input, and tended to assume a long press was needed rather than a short tap. The short delay between a successful input and the appearance of the next screen also caused confusion, and resulted in errors being made by people tapping the same spot twice, in the belief that their first tap hadn't registered. This observation led us to introduce a short waiting animation to indicate successful input.

One of the most interesting interaction challenges we observed was the interpretation of decision tree icons. Some icons were intended to be interpreted literally, others represented categories. In Western societies it is common to use an example to refer to a category (e.g. an apple to represent the category "fruit"). Yet during our field tests it became clear that category examples were often interpreted literally. This may be partially explained by the fact that no visual clues were given to indicate that a depicted item represented a category rather than just its literal self. However, when told certain images represented categories participants expressed a desire for exhaustive descriptions (e.g. adding more fruit examples to the fruit icon), which is problematic on small screens. Interestingly icons that used metaphors rather than examples to describe a category (e.g. a syringe to represent medicinal flora), seemed to work much better.

We undertook initial structured usability experiments with non-literate users who had been present during trainings. We designed a set of tasks of different difficulty levels intended to evaluate efficiency, accuracy and recall. However, conducting experiments in this environment presented multiple challenges. Individual evaluations were experienced as rude and awkward for members of such highly cooperative and communal cultures. Stopping people from helping those being evaluated was tricky, and not attempted after one minute of a user struggling with a task. For some, the tasks were so abstract and the context so intimidating that they were unable to perform. We allowed them help in order to avoid them feeling embarrassed. The results of the experiments were less encouraging than we had expected, with participants often performing poorly in terms of efficiency and accuracy. However, participants had received only limited training for 3 hours at most, some had not participated actively, and the unusual context experienced during evaluation made poor performers out of some who had been adept in the mapping exercise. We found that those who received training over multiple session performed much better, indicating the importance of repetition and duration of training.

\section{Conclusion}

This paper introduces our efforts to extend the reach of citizen science to communities hitherto excluded yet with vital contributions to make to improve both environmental and human well-being. By doing so, we believe that we open up new possibilities to address large complex problems collaboratively. The name 'Extreme Citizen Science' reflects these aspirations by focussing our efforts on developing ways to engage marginalised communities in contexts that are challenging due to their geographical, cultural, political, educational and socioeconomic situation.

By developing tools and methodologies specifically designed to adapt to diverse local contexts, and be accessible to users with widely varying levels of literacy and technical ability, the ExCiteS group aims to give any community the opportunity to participate in defining their problems, developing data collection interfaces and protocols to document them, then collecting the data and monitoring it over time. By analysing the data they collect and by building advocacy partnerships and strategies to support them to act on their findings, an increasing range of communities can use participatory citizen science to address issues that matter to them.

While still works in progress, our methodologies and the Sapelli platform provide a solid basis for further developments. By testing our approach in a range of different environments and among very different cultures we hope that our tools and methodologies will eventually be useful to any community no matter where they live or what threats they face.

The Sapelli platform has a number of unique features that meet the data collection and transmission requirements of our current and future projects. However it is generic enough to be applied in a wide variety of data collection scenarios - not necessarily only those that can be considered participatory citizen 
science initiatives. It is available now for anyone to try out. Some planned future extensions include support for video recording, a Web-interface for survey design, project management, and data querying, analysis and visualisation. As the platform matures we intend to release it under an open source license and form a developer community around it.

Finally, with the data collection and transmission elements of our platform advancing, the next challenge is to develop visualisation, analysis and editing approaches in ways intelligible to non-literate or technically and map illiterate users.

\section{ACKNOWLEDGEMENTS}

The authors are supported by the 'Extreme' Citizen Science - ExCiteS grant, funded by the Engineering and Physical Sciences Research Council (EPSRC reference $\mathrm{EP} / \mathrm{I} 025278 / 1)$.

\section{REFERENCES}

[1] Ehrlich and Ehrlich, "Solving the human predicament," International Journal of Environmental Studies, vol. 69 , no. 4 , pp. 557-565, 2012. DOI: $10.1080 /$ 00207233.2012.693281.

[2] Lewis and Nkuintchua, "Accessible technologies and FPIC: independent monitoring with forest communities in Cameroon," Participatory Learning and Action, vol. 65, pp. 151-165, 2012.

[3] Lewis, "Technological Leap-frogging in the Congo Basin, Pygmies and Global Positioning Systems in Central Africa: What has happened and where is it going?" African study monographs, vol. 43, pp. 1544, 2012.

[4] Muller, "Participatory Design: The Third Space in HCI," in Human-Computer Interaction: Development Process, Sears and Jacko, Eds., CRC Press, 2009, pp. 165-186.

[5] Bonney et al., Public Participation in Scientific Research: Defining the Field and Assessing Its Potential for Informal Science Education. CAISE, 2009. [Online]. Available: http:/ / eric.ed.gov/?id=ED519688.

[6] Silvertown, "A new dawn for citizen science," Trends in Ecology \& Evolution, vol. 24, no. 9, pp. 467-471, 2009. DOI: 10.1016/j.tree.2009.03.017.

[7] Goodchild, "Citizens as sensors: the world of volunteered geography," GeoJournal, vol. 69, no. 4, pp. 211-221, 2007. DOI: 10.1007/s10708-007-9111y.

[8] Roy et al., Understanding Citizen Science and Environmental Monitoring. NERC and NHM, 2012.

[9] Unwin, Ed., ICT4D: Information and Communication Technology for Development. Cambridge University Press, 2009.
[10] Chambers, "The origins and practice of participatory rural appraisal," World development, vol. 22, no. 7, pp. 953-969, 1994. DOI: 10.1016 / 0305 750X(94)90141-4.

[11] Sieber, "Public Participation Geographic Information Systems: A Literature Review and Framework," Annals of the Association of American Geographers, vol. 96 , no. 3, pp. 491-507, 2006. DOI: 10.1111/j.1467-8306.2006.00702.x.

[12] Anokwa et al., "Open Source Data Collection in the Developing World," Computer, vol. 42, no. 10, pp. 97-99, 2009. DOI: 10.1109/MC.2009.328.

[13] Vitos et al., "Making local knowledge matter, Supporting non-literate people to monitor poaching in Congo," in DEV'13 (January 11-12, 2013, Bangalore). DOI: $10.1145 / 2442882.2442884$.

[14] Aanensen et al., "EpiCollect: Linking Smartphones to Web Applications for Epidemiology, Ecology and Community Data Collection," PLOS ONE, vol. 4, no. 9, e6968, 2009. DOI: $10.1371 /$ journal.pone. 0006968 .

[15] Medhi et al., "Some Evidence for the Impact of Limited Education on Hierarchical User Interface Navigation," in CHI'13 (April 27-May 2, 2013, Paris), pp. 2813-2822. DOI: 10 . 1145 / 2470654. 2481390. 

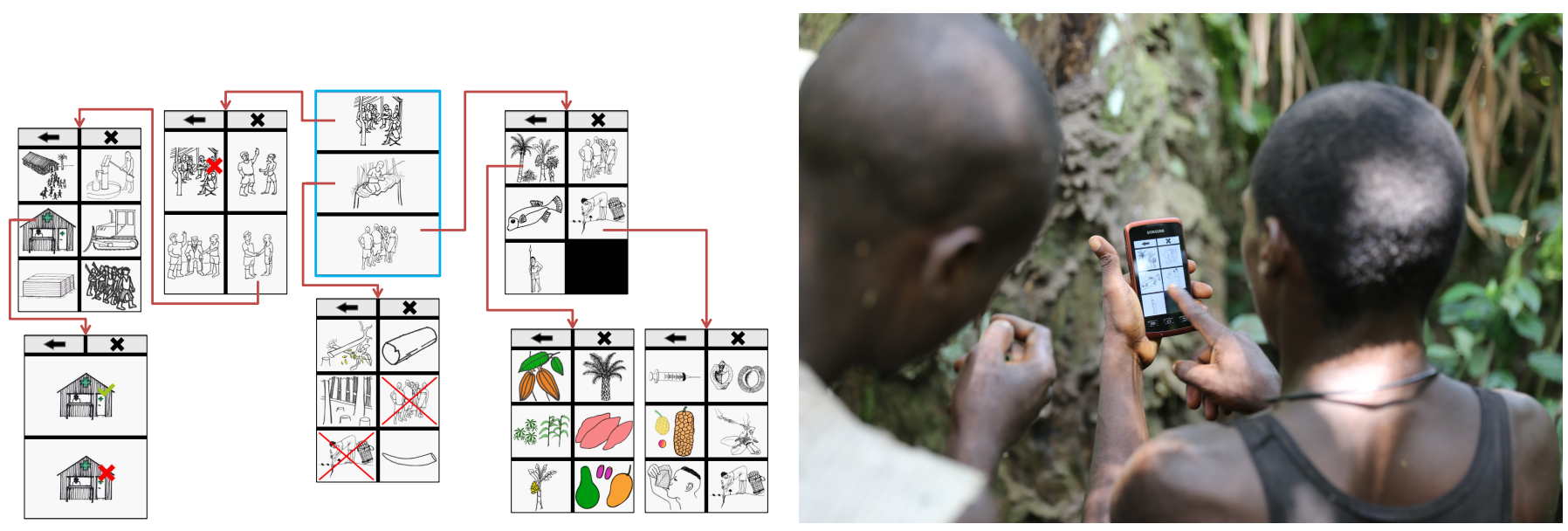

Fig. 1. On the left, part of the decision tree designed in collaboration with Forests Monitor, OI-FLEG, and Congolese forest communities. On the right, the tool being used in the forest 


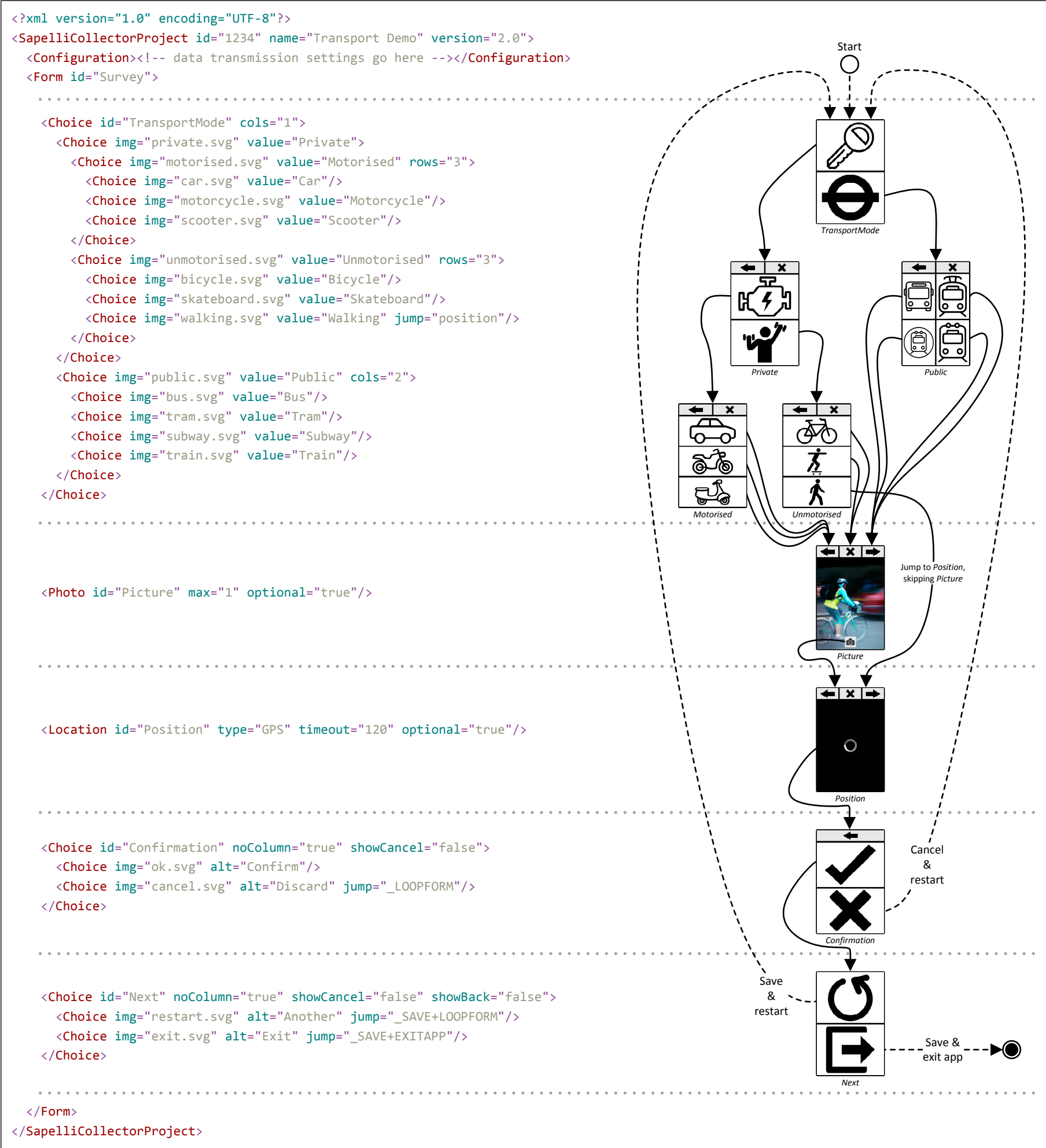

Fig. 2. On the left, a Sapelli XML description a survey about transport modes. On the right, a flow diagram illustrating how the survey will appear on the screen. The definition and appearance of each individual field is demarcated. 